\title{
Purely subjective variational preferences
}

DOI:

10.1007/s00199-016-1003-2

\section{Document Version}

Accepted author manuscript

Link to publication record in Manchester Research Explorer

\section{Citation for published version (APA):}

Webb, C. (2016). Purely subjective variational preferences. Economic Theory, 64, 121-137.

https://doi.org/10.1007/s00199-016-1003-2

\section{Published in:}

Economic Theory

\section{Citing this paper}

Please note that where the full-text provided on Manchester Research Explorer is the Author Accepted Manuscript or Proof version this may differ from the final Published version. If citing, it is advised that you check and use the publisher's definitive version.

\section{General rights}

Copyright and moral rights for the publications made accessible in the Research Explorer are retained by the authors and/or other copyright owners and it is a condition of accessing publications that users recognise and abide by the legal requirements associated with these rights.

\section{Takedown policy}

If you believe that this document breaches copyright please refer to the University of Manchester's Takedown Procedures [http://man.ac.uk/04Y6Bo] or contact uml.scholarlycommunications@manchester.ac.uk providing relevant details, so we can investigate your claim.

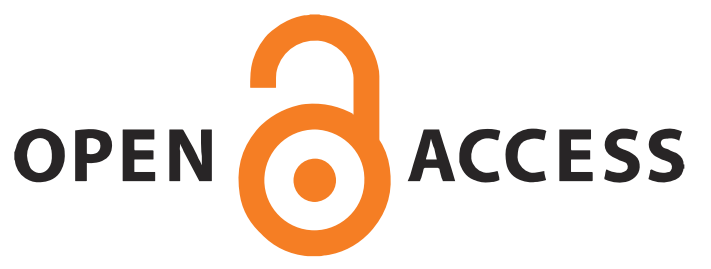




\title{
Purely Subjective Variational Preferences.
}

\author{
Craig S. Webb* \\ September 19, 2016
}

\begin{abstract}
Variational preferences (Maccheroni, Marinacci and Rustichini, 2006) are an important class of ambiguity averse preferences, compatible with Ellsberg-type phenomena. In this paper, a new foundation for variational preferences is derived in a framework of two-stages of purely subjective uncertainty. A similar foundation is obtained for purely subjective maxmin expected utility (Gilboa and Schmeidler, 1989). By establishing their axiomatic foundations without the use of extraneous probabilities, the conceptual appeal and applicability of these ambiguity models is enhanced.
\end{abstract}

Keywords: Variational preferences, ambiguity aversion, subjective uncertainty.

JEL Classification: D81

\section{Introduction}

Despite being inconsistent with subjective expected utility, the uncertainty averse pattern of preferences suggested by Ellsberg (1961) has been defended normatively (Gilboa, Postlewaite and Schmeidler, 2012; Gilboa, 2014) and descriptively (Camerer and Weber, 1992;

*Address: Economics, School of Social Sciences, The University of Manchester, England. Contact craig.webb@manchester.ac.uk. 
Fox and Tversky, 1995; Halevy, 2007). Many new theories have emerged to accommodate such behaviour (Gilboa and Marinacci, 2013). An important class of uncertainty averse preferences are the variational preferences of Maccheroni, Marinacci and Rustichini (2006), MMR hereforth. Variational preferences generalise the maxmin-expected utility (MEU) model of Gilboa and Schmeider (1989). The main theorem of this paper characterises a fully subjective version of variational preferences. The special case of purely subjective MEU preferences is also characterised.

In this paper, we consider preferences over acts with two stages of subjective uncertainty. As with Savage (1954), we employ a rich set of states and allow the outcome set to be arbitrary. Ellsberg-type preferences, in our case, refers to being more uncertainty averse towards first-stage uncertainty than second-stage uncertainty. That is, we assume source dependence of uncertainty attitudes (Heath and Tversky, 1991; Fox and Tversky, 1995; Chow and Sarin, 2001; Wakker, 2001; Abdellaoui et al. 2011), also called issue preference (Ergin and Gul, 2009; Strzalecki, 2011). Indeed, we will assume that, while second-stage uncertainty can be subjective, the decision maker displays no particular aversion to such uncertainty. The two-stage framework is used to develop a subjective notion of averaging events and averaging acts. Ellsberg's example corresponds to a decision maker who prefers first-stage uncertainty to be averaged out. We call such a preference second-stage uncertainty aversion. It will be shown that, when combined with other known axioms, second-stage uncertainty aversion characterises purely subjective variational preferences.

To the best of my knowledge, there has been no purely subjective foundation for variational preferences. For MEU, there has been more success, although a foundation at the full level of generality of Savage (1954) remains elusive. Chateauneuf (1991) assumed a single stage of subjective uncertainty and derived MEU with linear utility for final outcomes. MMR's axioms could be similarly reinterpreted. Recent derivations of MEU have used a single stage of purely subjective uncertainty and assumed topological conditions on the final outcome set (Casadesus-Masanell, Klibanoff and Ozdenoren, 2000; Ghirardato, 
Maccheroni, Marinacci and Siniscalchi, 2003; Alon and Schmeidler, 2014). A particular benefit of this approach is that, unlike the approach taken in this paper, it allows for finite state spaces. The topological richness of the outcome set facilitates the derivation of cardinal utility and separation of decision weights from utility, yielding a biseparable representation (Ghirardato and Marinacci, 2001). Variational preferences are not necessarily biseparable, hence such approaches have not yielded new foundations for variational preferences. This paper advances the literature by characterising purely subjective variational preferences in a different, special case of the Savage (1954) framework.

\section{Ellsberg and Multiple Stages of Uncertainty}

The acts considered in Ellsberg (1961) can be naturally viewed as having two separate stages of uncertainty (Segal, 1987; Klibanoff, Marinacci and Mukerji, 2005; Nau, 2006; Halevy, 2007; Chew and Sagi, 2008; Ergin and Gul, 2009; Seo, 2009; Machina, 2011). A simple version of Ellsberg's three-colour problem imagines a ball will be chosen from an urn containing three numbered balls. Ball 1 is red, and the remaining balls are either yellow or black. In the first stage of uncertainty, the composition of the urn, a colour for each remaining ball, is determined. In the second stage, the number of the ball is determined. Hence, the outcome of choices in this problem are determined by the realisation of two states, $s$ and $t$, where $s \in\{Y Y, Y B, B Y, B B\}$ and $t \in\{1,2,3\}$. Consider the following choices: 


\section{Choice One.}

$$
\begin{aligned}
& f \text { pays } £ 100 \text { only if red. } v^{\prime} s \quad g \text { pays } £ 100 \text { only if yellow. }
\end{aligned}
$$

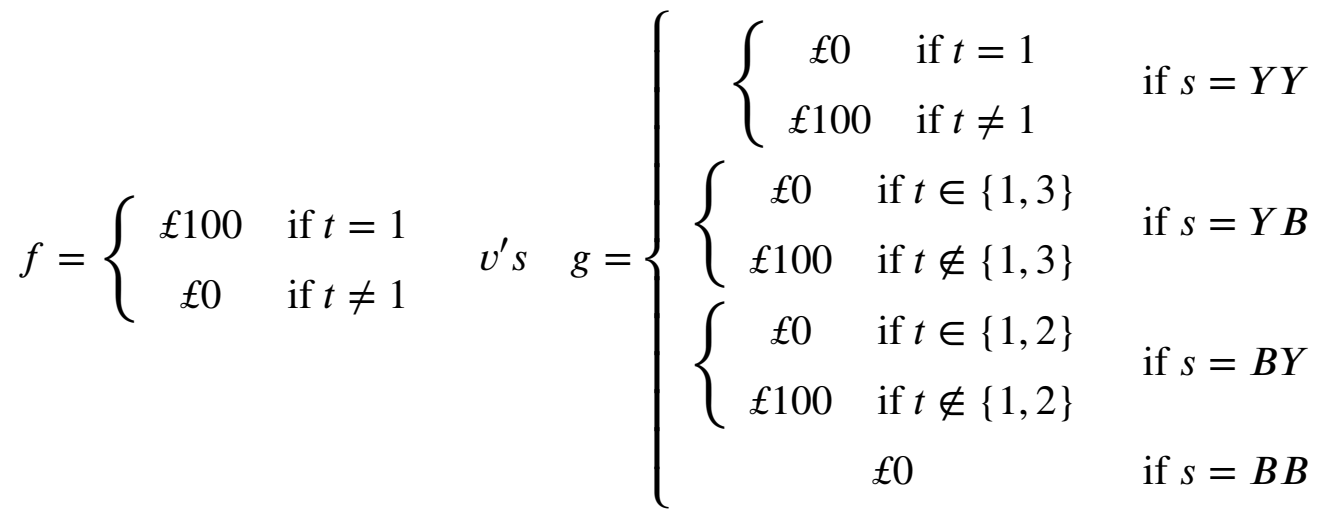

\section{Choice Two.}

$\tilde{f}$ pays $£ 100$ only if a red or black. $\quad v^{\prime} s \quad \tilde{g}$ pays $£ 100$ only if a yellow or black.

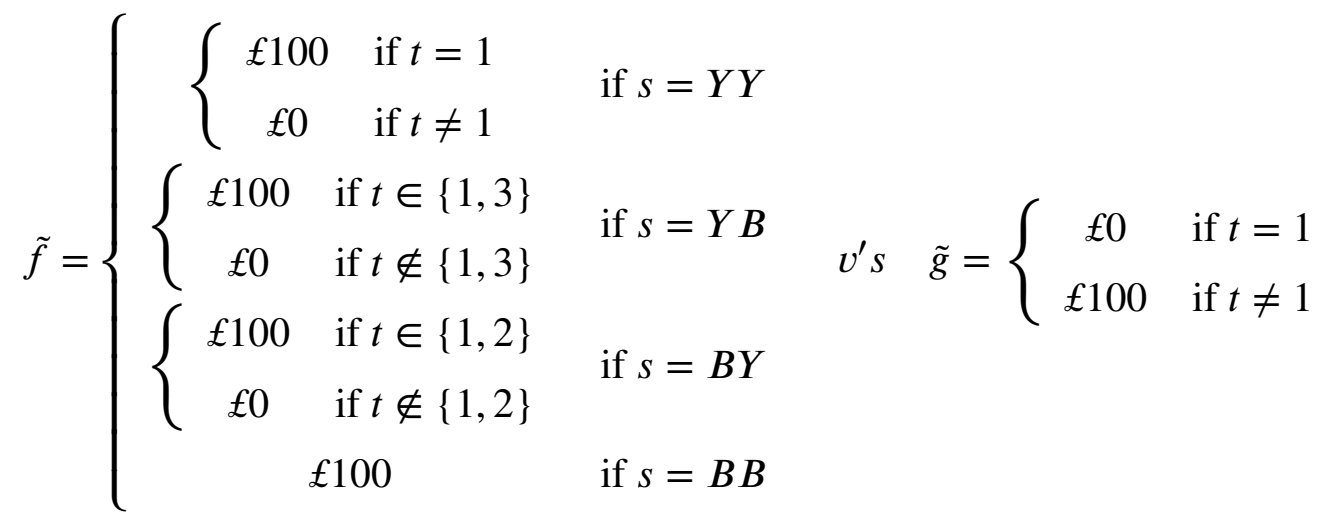

The modal pattern of preferences seems to be $f$ preferred to $g$ and $\tilde{g}$ preferred to $\tilde{f}$. The above presentation makes it clear that the commonly preferred acts, $f$ and $\tilde{g}$, have no firststage uncertainty. For some reason, the acts with uncertainty about the second-stage un- 
certainty are disliked. In the following section, the two-stage framework is formalised so that, in Section 6 this aversion to uncertainty about second-stage uncertainty can be precisely defined. When combined with known axioms, this will provide the key axiom for variational preferences in this framework.

\section{The Two-Stage Framework}

Let $\mathcal{S}$ denote a finite set of first-stage states with $\sigma$-algebra $\mathcal{E}_{S}$. Let $\mathcal{T}$ denote a set of secondstage states with $\sigma$-algebra $\mathcal{E}_{\mathcal{T}}$. Let $\mathcal{X}$ denote a finite set of outcomes. ${ }^{1}$ We impose richness on the second-stage state space $\mathcal{T}$ in what follows via a structural condition (second-stage solvability). Let $\mathcal{E}_{S} \otimes \mathcal{E}_{\mathcal{T}}=\sigma\left\{A \times B: A \in \mathcal{E}_{S}, B \in \mathcal{E}_{\mathcal{T}}\right\}$. An act is a function $f$ : $S \times \mathcal{T} \rightarrow \mathcal{X}$ that is measurable with respect to $\mathcal{E}_{S} \otimes \mathcal{E}_{\mathcal{T}}$. The set of acts is $\mathcal{A}$ and contains all such functions. The decision maker is modelled using a preference relation $\geqslant$, a binary relation defined over $\mathcal{A}$.

If $A \in \mathcal{E}_{S}$ is a first-stage event, then $f_{A} g$ refers to the act such that $f_{A} g(s, \cdot)=f(s, \cdot)$ if $s \in A$ and $f_{A} g(s, \cdot)=g(s, \cdot)$ otherwise. If $B \in \mathcal{E}_{\mathcal{T}}$ is a second-stage event, then $f_{B} g$ refers to the act such that $f_{B} g(\cdot, s)=f(\cdot, t)$ if $t \in B$ and $f_{B} g(\cdot, t)=g(\cdot, t)$ otherwise. An act $f$ is constant if there is $x \in \mathcal{X}$ such that, for all $s \in S$ and all $t \in \mathcal{T}, f(s, t)=x$. We abuse notation and write $x$ to mean the constant act yielding $x$. An event $A \in \mathcal{E}_{i}, i \in\{\mathcal{S}, \mathcal{T}\}$, is null if $f_{A} h \sim g_{A} h$ for all $f, g, h \in \mathcal{A}$, otherwise it is non-null.

Given an act $f: S \times \mathcal{T} \rightarrow \mathcal{X}$ and first-stage state $s \in \mathcal{S}$, we define the second-stage act $f_{s}: \mathcal{T} \rightarrow \mathcal{X}$ such that $f_{s}(t)=f(s, t)$ for all $t \in \mathcal{T}$. An act $f$ is second-stage constant if $f_{s}$ is constant for all $s \in S$. An act $f$ is first-stage constant if, for all $s, \tilde{s} \in \mathcal{S}$, the second-stage acts $f_{s}$ and $f_{\tilde{s}}$ coincide. Preferences $\geqslant$ over first-stage constant acts naturally induce preferences over second-stage acts, that we also denote $\geqslant$, as follows: $f_{s} \geqslant g_{s}$ if

\footnotetext{
${ }^{1}$ All results can be extended to simple acts with $S$ and $\mathcal{X}$ arbitrary.
} 
and only if $h \geqslant \tilde{h}$, where $h$ and $\tilde{h}$ are first-stage constant acts with $h_{s}=f_{s}$ and $\tilde{h}_{s}=g_{s}$ for all $s \in S$.

Subjective expected utility evaluates acts as follows:

$$
f \mapsto \int_{\mathcal{S}} \int_{\mathcal{T}} u(f(s, t)) d p(s, t)
$$

where $p$ is a joint probability measure over $\mathcal{E}_{\mathcal{S}} \otimes \mathcal{E}_{\mathcal{T}}$, and $u: \mathcal{X} \rightarrow \mathbb{R}$ is a strictly $\geqslant-$ increasing utility function.

Denote by $\mathcal{P}_{S}$ the set of all finitely additive probability measures over the first-stage state space $\mathcal{S}$. A function $c: \mathcal{P}_{\mathcal{S}} \rightarrow[0, \infty]$ is grounded if its infimum value is zero. Preferences $\geqslant$ have a subjective variational representation if acts are evaluated as follows:

$$
f \mapsto \min _{p \in \mathcal{P}_{S}}\left(\int_{\mathcal{S}} \int_{\mathcal{T}} u(f(s, t)) d p(s) d q(t)+c(p)\right)
$$

where $q$ is a probability measure over second-stage events $\mathcal{E}_{\mathcal{T}}, u: \mathcal{X} \rightarrow \mathbb{R}$ is a strictly $\geqslant$ increasing utility function and $c: \mathcal{P}_{S} \rightarrow[0, \infty]$ is a grounded, convex, and lower semicontinuous function. The function $c$ is called the ambiguity index. In this paper, the probability measure $q$ is convex-valued: for all $\alpha \in[0,1]$ there exists $A \in \mathcal{E}_{\mathcal{T}}$ such that $q(A)=\alpha$.

\section{Variational Preferences in the AA Framework}

The Anscombe-Aumann (AA) framework is the special case of our framework where $\mathcal{T}$ is already equipped with a convex-valued probability measure $q: \mathcal{E}_{\mathcal{T}} \rightarrow[0,1]$. Then, second-stage acts induce, via the probability measure $q$ on $\mathcal{T}$, lotteries on $\mathcal{X}$. Let $\Delta(\mathcal{X})$ be the set of lotteries over $\mathcal{X}$. Suppose the act $f$ takes values in $\left\{x_{1}, \ldots, x_{n}\right\}$. Then, the act $f$ 
can be identified with a function $F: S \rightarrow \Delta(\mathcal{X})$ such that:

$$
F(s)=\left(q\left(f_{s}^{-1}\left(x_{1}\right)\right), x_{1} ; \ldots ; q\left(f_{s}^{-1}\left(x_{n}\right)\right), x_{n}\right) \quad \text { for all } s \in \mathcal{S} \text {. }
$$

We call $F$ an $A A$ act, the set of which is $\mathcal{A}^{*}$. Preferences $\geqslant$ over $\mathcal{A}$ naturally induce preferences over $\mathcal{A}^{*}$. For all $F, G \in \mathcal{A}^{*}$, and $\alpha \in[0,1]$, define $\alpha F+(1-\alpha) G$ as the AA act that yields the well-defined lottery $\alpha F(s)+(1-\alpha) G(s)$, for all $s \in \mathcal{S}$. The set of AA acts $\mathcal{A}^{*}$ can then be taken to be a convex set. The key axioms for variational preferences, weak certainty independence and uncertainty aversion, and the continuity axiom used, all make explicit use of the convexity of the set of acts.

Axiom MMR1 (Weak Ordering): Preferences $\geqslant$ over $\mathcal{A}^{*}$ are complete and transitive. Axiom MMR2 (Weak Certainty Independence): For all $F, G \in \mathcal{A}^{*}$, lotteries $P, Q \in$ $\Delta(\mathcal{X})$, and $\alpha \in(0,1): \alpha F+(1-\alpha) P \geqslant \alpha G+(1-\alpha) P$ only if $\alpha F+(1-\alpha) Q \geqslant \alpha G+(1-\alpha) Q$ Axiom MMR3 (Mixture Continuity): For all $F, G, H \in \mathcal{A}^{*},\{\alpha \in[0,1]: \alpha F+(1-$ $\alpha) G \geqslant H\}$ and $\{\alpha \in[0,1]: H \geqslant \alpha F+(1-\alpha) G\}$ are closed subsets of the $[0,1]$ interval. Axiom MMR4 (Monotonicity): $F(s) \geqslant G(s)$ for all $s \in S$ only if $F \geqslant G$.

Axiom MMR5 (Uncertainty Aversion): For all $F, G \in \mathcal{A}^{*}$ and $\alpha \in(0,1): F \sim G$ only if $\alpha F+(1-\alpha) G \geqslant F$.

Axiom MMR6 (Nondegeneracy): $F>G$ for some $F, G \in \mathcal{A}^{*}$.

Preferences have a variational representation in the AA framework if there exists a nonconstant affine function $U: \Delta(\mathcal{X}) \rightarrow \mathbb{R}$ and a grounded, convex, and lower semicontinuous function $c: \mathcal{P}_{S} \rightarrow[0, \infty]$ such that, for all $F, G \in \mathcal{A}^{*}$ :

$$
F \geqslant G \Leftrightarrow \min _{p \in \mathcal{P}_{S}}\left(\int_{S} U(F) d p+c(p)\right) \geqslant \min _{p \in \mathcal{P}_{S}}\left(\int_{S} U(G) d p+c(p)\right) .
$$

The following theorem characterises variational preferences in the AA framework: 
Theorem 4.1 (Maccheroni, Marinacci \& Rustichini, 2006). Preferences $\geqslant$ over AnscombeAumann acts $\mathcal{A}^{*}$ satisfy axioms MMRl-6 (weak ordering, weak certainty independence, mixture continuity, monotonicity, uncertainty aversion, and nondegeneracy) if and only if they admit a variational representation. In such a representation, utility $U$ is cardinal and the ambiguity index $c$ is a ratio scale.

\section{Second-Stage Subjective Expected Utility}

Consider the following axioms for preferences $\geqslant$ over $\mathcal{A}$ :

Axiom 1 (Weak Ordering): Preferences $\geqslant$ over $\mathcal{A}$ are complete and transitive.

Axiom 2 (Monotonicity): $f(s, t) \geqslant g(s, t)$ for all $s \in \mathcal{S}$ and $t \in \mathcal{T}$ only if $f \geqslant g$.

Axiom 3 (Non-Degeneracy): There exist constant acts such that $x>y$.

Axiom 4 (Weak Comparative Probability): For all events $A, B \in \mathcal{E}_{\mathcal{T}}$ and outcomes $x, \tilde{x}, y, \tilde{y} \in \mathcal{X}$, with $x>y$ and $\tilde{x}>\tilde{y}, x_{A} y \geqslant x_{B} y$ only if $\tilde{x}_{A} \tilde{y} \geqslant \tilde{x}_{B} \tilde{y}$.

Axiom 5 (Archimedeanity): If $S=A^{1}, A^{2}, \ldots$, is a sequence of non-null second-stage events such that $x_{A^{1}} f>x_{A^{1}} g$ and $x_{A^{i}} f \sim x_{A^{i+1}} g$ for all $i=1,2, \ldots$, then $S$ is finite.

Apart from the fact that we have adapted these axioms to two-stage uncertainty, axioms 1-5 are well-known. Rather than use a second-stage version of Savage's P6, small event continuity, we use an Archimedean axiom and a solvability assumption, as follows:

Structural Assumption (Second-Stage Solvability): For all $f \in \mathcal{A}$ and $x, y \in \mathcal{X}$, with $x \geqslant f \geqslant y$, there exists $A \in \mathcal{E}_{\mathcal{T}}$ such that $f \sim x_{A} y$.

In this way, non-necessary conditions are separated from the axiom set. Subjective variational preferences necessarily satisfy axioms 1-5. The following axiom, the second-stage 
sure-thing principle plays a similar role here to that played by the weak certainty independence axiom in the AA framework. Strzalecki (2011) first introduced this axiom. ${ }^{2}$

Axiom 6 (Second-Stage Sure-Thing Principle): For all acts $f, g \in \mathcal{A}$, first-stage constant acts $h, \tilde{h} \in \mathcal{A}$, and second-stage events $E \in \mathcal{E}_{\mathcal{T}}: f_{E} h \geqslant g_{E} h$ only if $f_{E} \tilde{h} \geqslant g_{E} \tilde{h}$.

For $c \in \mathbb{R}$, let $\mathbf{c}=(c, \ldots, c) \in \mathbb{R}^{n}$. A function $\phi: \mathbb{R}^{n} \rightarrow \mathbb{R}$ is vertically invariant if for all $x \in \mathbb{R}^{n}, \alpha \in[0,1], \phi(\alpha x+(1-\alpha) \mathbf{c})=\phi(\alpha x)+(1-\alpha) c$. For finite $S=\left\{s_{1}, \ldots, s_{n}\right\}$, preferences are represented by invariant second-stage expected utility if acts are evaluated as follows:

$$
f \mapsto \phi\left(\int_{\mathcal{T}} u\left(f\left(s_{1}, t\right)\right) d q(t), \ldots, \int_{\mathcal{T}} u\left(f\left(s_{n}, t\right)\right) d q(t)\right)
$$

where $\phi: \mathbb{R}^{n} \rightarrow \mathbb{R}$ is monotonic, mixture-continuous, and vertically invariant, $q$ is a convex-valued probability measure over $\mathcal{E}_{\mathcal{T}}$, and $u: \mathcal{X} \rightarrow \mathbb{R}$ is a strictly $\geqslant$-increasing utility function. This class of preferences encompasses subjective variational preferences. Axioms 1-6 characterise invariant second-stage expected utility:

Theorem 1. Assume second-stage solvability. Then, preferences $\geqslant$ over $\mathcal{A}$ satisfy axioms 1-6 (weak ordering, monotonicity, nondegeneracy, weak comparative probability, Archimedeanity, the second-stage sure-thing principle) if and only if they admit an invariant second-stage expected utility representation. In such a representation, utility $u$ is cardinal and the probability measure $q$ is unique.

\section{Averaging Events and Acts}

The uncertainty aversion axiom captures a preference for hedging subjective uncertainty. If $f \sim g$ holds, the mixture $\frac{1}{2} f+\frac{1}{2} g$ smooths out the subjective uncertainty of each act. Here, we construct a similar idea using only subjective uncertainty. The approach taken here is admittedly less straightforward. We will use the notion of a second-stage event average.

\footnotetext{
${ }^{2}$ There it is called Weak $\mathcal{F}_{a}$ Independence.
} 
A second-stage event average of two events $A$ and $C$ will, intuitively, correspond to an event $B$ that evenly mixes the probabilities of $A$ and $C$. To visualise the idea, consider the following example. Imagine a container of different coloured rice (or similar fine material). There is red, yellow, and possibly other coloured rice. Bets will be placed on the colour of a grain selected from the mixed bucket. Event $A$ is "red" and event $C$ is "yellow". Suppose that:

$$
\left\{\begin{array}{cc}
£ 100 & \text { if } s \in A \\
£ 0 & \text { if } s \notin A
\end{array}>\left\{\begin{array}{cl}
£ 100 & \text { if } s \in C \\
£ 0 & \text { if } s \notin C
\end{array}\right.\right.
$$

The interpretation is that the decision believes there are more red grains in the mix than yellow. Abdellaoui and Wakker (2005) refer to $A$ being revealed as more likely than $C$ in a basic sense. Now suppose we can scoop out a subset $E$ of red grains and add the same amount of yellow grains back. Then, "red" is the event $A \backslash E$ and "yellow" is the event $C \cup E$. Suppose that, perhaps by iterating the procedure, we elicit the following indifference:

$$
\left\{\begin{array} { c l } 
{ £ 1 0 0 } & { \text { if } s \in A \backslash E } \\
{ £ 0 } & { \text { if } s \notin A \backslash E }
\end{array} \sim \left\{\begin{array}{cc}
£ 100 & \text { if } s \in C \cup E \\
£ 0 & \text { if } s \notin C \cup E
\end{array}\right.\right.
$$

Then, $A \backslash E$ is revealed equally likely to $C \cup E$, in a basic sense. We call any event $B$ that is also revealed equally likely to $A \backslash E$ and $C \cup E$ an event average of $A$ and $C$. This a subjective definition of averaging events. Applying this idea in the two-stage framework leads to the following:

Definition (Second-Stage Event Average): Given outcomes $x, y \in \mathcal{X}$ with $x>y$ and second-stage events $A, B, C \in \mathcal{E}_{\mathcal{T}}, B$ is a second-stage event average of $A$ and $C$ if $x_{A} y \geqslant$ $x_{C} y$ and there exists $E \subset A \backslash C$ such that $x_{B} y \sim x_{A \backslash E} y \sim x_{C \cup E} y$.

We also call $B$ a second-stage event average of $A$ and $C$ if it is a second-stage event average of $C$ and $A$. The following lemma further justifies this terminology:

Lemma 6.1. For subjective variational preferences, $B \in \mathcal{E}_{\mathcal{T}}$ is a second-stage event average of $A$ and $B$ only if $q(B)=\frac{1}{2} q(A)+\frac{1}{2} q(C)$. 
To see this, suppose that $B$ a second-stage event average of $A$ and $C$, and that preferences admit a subjective variational preferences representation. Then, $x_{B} y \sim x_{A \backslash E} y$ holds if and only if:

$$
q(B) u(x)+(1-q(B)) u(y)=q(A \backslash E) u(x)+(1-q(A \backslash E)) u(y),
$$

and $x_{B} y \sim x_{C \cup E} y$ holds if and only if,

$$
q(B) u(x)+(1-q(B)) u(y)=q(C \cup E) u(x)+(1-q(C \cup E)) u(y) .
$$

Given $u(x)>u(y)$ and $E \subset A \backslash C$, these are jointly equivalent to:

$$
\begin{gathered}
q(B)=q(A \backslash E)=q(C \cup E) \\
\Leftrightarrow q(B)=q(A)-q(E)=q(C)+q(E) \\
\Leftrightarrow q(A)-q(B)=q(B)-q(C) \\
\Leftrightarrow q(B)=\frac{1}{2} q(A)+\frac{1}{2} q(C) .
\end{gathered}
$$

Using the notion of a second-stage event average, a notion of averaging acts can be constructed. For two acts $f$ and $g$, in each first-stage state $s$, replace the second-stage acts $f_{s}$ and $g_{s}$ with equivalent binary acts $x_{A(s)} y$ and $x_{C(s)} y$, then construct an act with second-stage acts $\left(x_{B(s)} y\right)_{s}$ such that each $B(s)$ is a second-stage event average of $A(s)$ and $C(s)$ :

Definition (Second-Stage Act Average): Given acts $f, g, h \in \mathcal{A}$ and outcomes $x, y \in \mathcal{X}$ with $x>y$ and $x \geqslant j \geqslant y$ for $j=f, g, h$ and, for all $s \in S$ :

$$
f_{s} \sim\left(x_{A(s)} y\right)_{s} \quad \text { and } \quad g_{s} \sim\left(x_{B(s)} y\right)_{s} \quad \text { and } \quad h_{s} \sim\left(x_{C(s)} y\right)_{s},
$$

where $A(s), B(s), C(s) \in \mathcal{E}_{\mathcal{T}}, g$ is a second-stage act average of $f$ and $h$ if, for all $s \in S$, $B(s)$ is a second-stage event average of $A(s)$ and $C(s)$.

This definition of averaging acts is purely subjective. It would be problematic if it de- 
pended on the particular $x$ and $y$ outcomes, but our axiom set rules out such a dependence. Implementing the definition could, depending on the acts under consideration, require the elicitation a large number of indifferences. The following lemma justifies the term secondstage act average:

Lemma 6.2. For subjective variational preferences, $g$ is a second-stage act average of $f$ and $h$ if and only if, for all $s \in \mathcal{S}$,

$$
\int_{\mathcal{T}} u(g(s, t)) d q(t)=\frac{1}{2} \int_{\mathcal{T}} u(f(s, t)) d q(t)+\frac{1}{2} \int_{\mathcal{T}} u(h(s, t)) d q(t) .
$$

The following axiom uses the above notion of second-stage act averages to define a subjective version of the uncertainty aversion axiom:

Axiom 7 (Second-Stage Uncertainty Aversion): For all acts $f, g, h \in \mathcal{A}$, with $g$ a secondstage act average of $f$ and $h, f \sim h$ only if $g \geqslant f$.

Let us reconsider the Ellsberg three-colour problem, interpreted in the two-stage framework. There are three numbered balls in an urn. Ball 1 is red, balls 2 an 3 are known only to be yellow or black. The first stage of uncertainty concerns the unknown colours $\{Y Y, Y B, B Y, B B\}$ and, in the second stage, a number is chosen from $\{1,2,3\}$. Let $x>y$. The act $g$, that pays $x$ only if a yellow ball is drawn, is typically considered worse than the act $f$, that pays $x$ only if a red ball is drawn. Intuitively, one of the three balls is red, while it is possible that none of the other balls are yellow. The same reasoning suggests that $f$ would be preferred to an act $g^{*}$, that pays $x$ only if a black ball is drawn. Further, appealing 
to the symmetry of their descriptions, $g \sim g^{*}$ is reasonable, and we assume this holds:

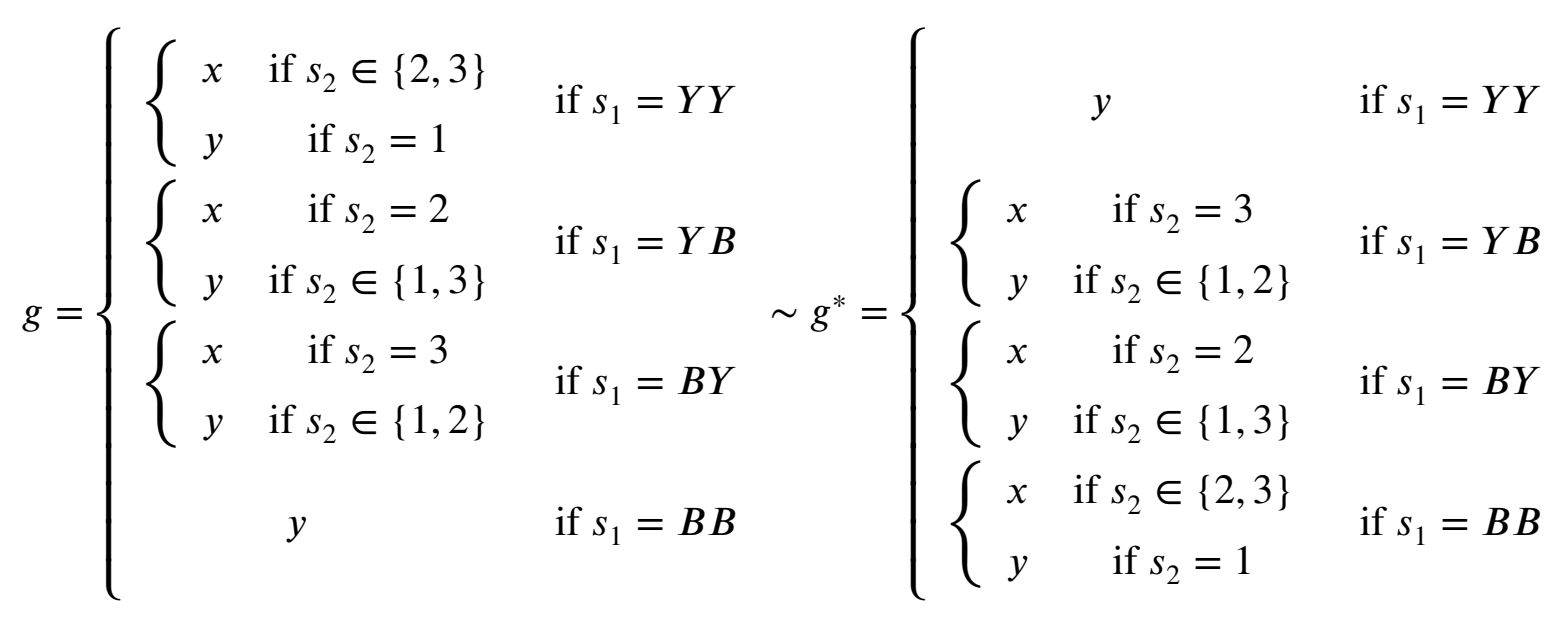

We appeal to symmetry again and assume:

$$
x_{\{1\}} y \sim x_{\{2\}} y \sim x_{\{3\}} y \quad \text { and } \quad x_{\{1,2\}} y \sim x_{\{1,3\}} y \sim x_{\{2,3\}} y
$$

Hence, $\{1\}$ is a second-stage event average of $\{2,3\}$ and $\emptyset$. Also, $\{1\}$ is a second-stage event average of $\{2\}$ and $\{3\}$. It follows that $x_{\{1\}} y=f$ is a second-stage act average of $g$ and $g^{*}$, therefore $f>g$ is consistent with second-stage uncertainty aversion. A similar argument can be used to argue that $\tilde{g}>\tilde{f}$ is simultaneously consistent with secondstage uncertainty aversion. Second-stage uncertainty averse preferences are consistent with Ellberg's example. We now state the main theorem of this paper:

Theorem 2. Assume second-stage solvability. Then, preferences $\geqslant$ over $\mathcal{A}$ satisfy axioms 1-7 (weak ordering, monotonicity, nondegeneracy, weak comparative probability, Archimedeanity, the second-stage sure-thing principle and second-stage uncertainty aversion) if and only if they admit a subjective variational representation. In such a representation, utility $u$ is cardinal, the ambiguity index $c$ is a ratio scale, and the probability measure $q$ is unique.

Finally, consider the following restriction of axiom 7: 
Axiom 7* (Second-Stage Uncertainty Neutrality): For all acts $f, g, h \in \mathcal{A}$, with $g$ a second-stage act average of $f$ and $h, f \sim h$ only if $g \sim f$.

We have the following observation:

Observation 6.3. If, in statement 1 of Theorem, axiom A7 (second-stage uncertainty aversion) is replaced with axiom $A 7^{*}$ (second-stage uncertainty neutrality), then subjective expected utility holds.

\section{Second-Order Risk Aversion}

Strzalecki (2011), following Ergin and Gul (2009), referred to the following axiom as second-order risk aversion: ${ }^{3}$

Axiom $7^{* *}$ (Second-Order Risk Aversion): For all second-stage constant acts $f, g \in \mathcal{A}$, and second-stage events $A \in \mathcal{E}_{\mathcal{T}}, f \sim g$ only if $f_{A} g \geqslant f$.

Second-order risk aversion considers acts $f$ and $g$ that may have first-stage uncertainty, but no second-stage uncertainty. Considering the act $f_{A} g$, second-stage uncertainty is introduced, but the first-stage uncertainty is somewhat smoothed, with the overall change being preferable. Second-order risk aversion seems a more elegant axiom than secondstage uncertainty aversion. The following theorem implies that, if $\mathcal{X}$ is suitably solvable, then axiom 7 can be replaced with axiom $7^{* *}$ in Theorem 2:

Theorem 3. Assume preferences $\geqslant$ satisfy axioms 1-6 (weak ordering, monotonicity, nondegeneracy, weak comparative probability, Archimedeanity, and the second-stage surething principle), second-stage solvability holds, and all acts have certainty equivalents. Then, axiom 7 (second-stage uncertainty aversion) holds if and only if axiom $7^{* *}$ (secondorder risk aversion) holds.

\footnotetext{
${ }^{3}$ Translating the notation of that paper: $\Sigma_{a}$ amounts to our second-stage events $\mathcal{E}_{\mathcal{T}}$ and $\Sigma_{b}$ to our first-stage events $\mathcal{E}_{S}, \Sigma_{a}$-measurability is here called first-stage constant, and $\Sigma_{b}$-measurability is here called secondstage constant.
} 
Preferences $\geqslant$ have a second-order expected utility representation (Ergin and Gul, 2009) if acts are evaluated as follows:

$$
f \mapsto \int_{S} \psi\left(\int_{\mathcal{T}} u(f(s, t)) d q(t)\right) d p(s)
$$

where $p$ is a probability measure over first-stage events $\mathcal{E}_{S}, q$ is a probability measure over second-stage events $\mathcal{E}_{\mathcal{T}}, u: \mathcal{X} \rightarrow \mathbb{R}$ is a strictly $\geqslant$-increasing utility function and $\psi: \mathbb{R} \rightarrow \mathbb{R}$ is strictly increasing and continuous. Theorem 2 of Strzalecki (2011) gives the equivalence of concave $\psi$ and second-order risk aversion. Our theorem 3 shows the equivalence of concavity of the $\phi$ obtained in Theorem 1 and second-order risk aversion.

\section{Maxmin Expected Utility}

Preferences $\geqslant$ have a subjective maxmin expected utility (MEU) representation if acts are evaluated as follows:

$$
f \mapsto \min _{p \in C}\left(\int_{\mathcal{S}} \int_{\mathcal{T}} u(f(s, t)) d p(s) d q(t)\right)
$$

where $q$ is a convex-valued probability measure over second-stage events $\mathcal{E}_{\mathcal{T}}, u: \mathcal{X} \rightarrow \mathbb{R}$ is a strictly $\geqslant$-increasing utility function and $C \subseteq \mathcal{P}_{S}$ is a convex set of probability measures. They are a special case of variational preferences, seen by taking $c$ that is zero on $C$ and infinite outside of $C$. Hence, the axioms of Theorem 2 are necessary but not sufficient for MEU. An additional axiom is required, as follows:

Axiom 8 (Second-Stage Constant Independence): For all acts $f, \tilde{f}, g, \tilde{g} \in \mathcal{A}$, and outcome $x \in \mathcal{X}$, with $\tilde{f}$ a second-stage act average of $f$ and $x$ and $\tilde{g}$ a second-stage act average of $g$ and $x, f \sim g$ only if $\tilde{f} \sim \tilde{g}$.

We now state the following foundation for purely subjective maxmin expected utility: 
Theorem 4. Assume second-stage solvability. Then, preferences $\geqslant$ over $\mathcal{A}$ satisfy axioms 1-8 (weak ordering, monotonicity, nondegeneracy, weak comparative probability, Archimedeanity, the second-stage sure-thing principle, second-stage uncertainty aversion, and second-stage constant independence) if and only if they admit a subjective maxmin expected utility representation. In such a representation, utility $u$ is cardinal, the set of priors $C$ is unique, and the probability measure $q$ is unique.

\section{Discussion of Frameworks of Uncertainty}

The main results of this paper have been to characterise existing ambiguity models in a framework different to their original axiomatisations. This section discusses the frameworks commonly employed in modelling choice under uncertainty, to justify and clarify the contribution of this paper. Choice under uncertainty is now a vast field with various frameworks employed. Let us restrict attention to frameworks that employ, as primitives, a set of states $\mathcal{S}$, with $\sigma$-algebra of events $\mathcal{E}_{\mathcal{S}}$, and a set of outcomes $\mathcal{X}$. States and outcomes are primitives in the sense that, once these objects are understood, all other constructions can be made only in terms of these objects. For example, an act is a function from $S$ to $\mathcal{X}$, the set of acts is $\mathcal{A} \subseteq \mathcal{S}^{\mathcal{X}}$, a preference relation is a subset $\geqslant \subseteq \mathcal{S}^{\mathcal{X}} \times \mathcal{S}^{\mathcal{X}}$, and so on. The following frameworks are well-known:

The Savage Framework: Acts are measurable functions from $S$ to $\mathcal{X} . S$ has a rich structure (solvability). $\mathcal{X}$ is an arbitrary set.

The Wakker Framework: Acts are measurable functions from $S$ to $\mathcal{X}$. $S$ is an arbitrary set. $\mathcal{X}$ has a rich structure (topologically connectedness or algebraic solvability).

The Anscombe-Aumann Framework: Acts are measurable functions from $S$ to $\Delta(\mathcal{X})$. $\mathcal{S}$ is an arbitrary set. $\mathcal{X}$ is an arbitrary set. $\Delta(\mathcal{X})$ is the set of lotteries over $\mathcal{X}$.

The trade-off between the Savage and Wakker frameworks is quite clear. The Savage frame- 
work retains complete flexibility with the outcome set $\mathcal{X}$, hence theories developed in that framework are applicable for monetary outcomes, health outcomes, non-divisible goods, and so on. This price of this flexibility with $\mathcal{X}$ is the requirement that $S$ has a rich structure. ${ }^{4}$ Conversely, in the Wakker framework, the richness is bourne by $\mathcal{X}$, allowing for complete flexibility of the state space. Hence, the Wakker framework allows for monetary outcomes $(\mathcal{X}=\mathbb{R})$ and finitely many states, highly relevant for economic theory. Of course, requiring richness of $\mathcal{X}$ rules out applications to non-divisible goods, and so on.

In a sense, the Anscombe-Aumann (AA) framework retains the nicest properties of both the Savage and Wakker frameworks. The AA framework allows both $S$ and $\mathcal{X}$ to be arbitrary. However, the axiomatic derivation of expected utility, with cardinal utility and a unique subjective probability measure, requires additional structure. The AA framework's additional structure comes in two parts:

1. There are two stages of uncertainty.

2. The second stage of uncertainty is characterised by objective probabilities.

The additional structure imposed is very convenient. ${ }^{5}$ Axioms and proofs developed in the AA framework are more transparent. Clearly, however, this additional structure has some costs involved. Fishburn (1970:167) refers to the AA framework as one that employs extraneous probabilities. Ther term "extraneous" seems, at least to this author, to be carefully chosen. If one is describing the assumption that some objective probabilities are assumed, the more usual term would be "exogenous". Fishburn's use of the term "extraneous" seems to refer to both the fact the AA framework takes probabiliites as exogenously given, and the opinion that such objects are "surplus to requirement". Regarding the use of two stages,

\footnotetext{
${ }^{4}$ Certainly, in Savage's expected utility, $S$ must be infinite. It is not necessarily uncountable.

${ }^{5}$ Anscombe and Aumann (1962) actually assumed three stages of uncertainty, and reduced it to two stages. The presentation follows the modern interpretation. Also, the AA framework has also been used to describe the assumption simply that $\mathcal{X}$ is convex, without necessarily committing to the lottery interpretation. In this paper, we maintain the interpretation of a set of lotteries.
} 
while there are many occasions where multiple sources of uncertainty are present, it is a restrictive assumption. Trautmann and Wakker (2015) and others have criticised the use of backward induction implicit in the two-stage evaluation commonly used in the AA framework. Inspired in part by these concerns, further frameworks have been developed. Sarin and Wakker (1997), for example, cleverly avoided the use of two stage acts. Instead of uncertainty followed by risk, they assume uncertainty alongside risk, hence acts are either uncertain or are risky lotteries.

The Sarin and Wakker Framework: Acts are either measurable functions from $\mathcal{S}$ to $\mathcal{X}$, or elements of $\Delta(\mathcal{X}) . S$ is an arbitrary set. $\mathcal{X}$ is an arbitrary set.

Ergin and Gul (2009), and this paper, retain the two-stage feature of the AA framework, and all of its criticisms, but dispense with the requirement that the second stage of uncertainty is quantified by objective probabilities:

The Ergin and Gul Framework: Acts are measurable functions from $S \times \mathcal{T}$ to $\mathcal{X} . S$ and $\mathcal{T}$ have rich structures (solvability). $\mathcal{X}$ is an arbitrary set.

The specific two-stage formulation of the Ellsberg paradox used in Section 2 is an example of the Ergin and Gul framework. In this paper, we have assumed that both stages are subject to subjective uncertainty, but only the first stage is subject to ambiguity. That is, subjective uncertainty that cannot necessarily be quantified by a subjective probability measure. Uncertainty aversion here refers to the dislike of subjective uncertainty that the decision maker finds too "ambiguous" to confidently assign any unique probability. The distinction between first and second stage is made a priori, rather than revealed by preferences. This limitation has been discussed by Klibanoff, Marinacci and Mukerji (2005: 1872-5). A decision maker, when presented with the Ellsberg choices, may well entertain any belief about the composition, have second-order beliefs about such beliefs, have beliefs about the experiment in general, and so on. We have imposed a very specific set of assumptions. In certain applications the distinction can be quite clear, in particular if the decision maker has certain competences. For example, one might reasonably expect that an expert political 
commentator is more confident in his beliefs about the next US presidential election than his beliefs about the outcome of the Kentucky Derby. Of course, if the decision maker is a horse racing pundit, the converse is more reasonable. This connection between competence and ambiguity is supported by experimental evidence (Heath and Tversky, 1991; Fox and Tversky, 1995). Nevertheless, this remains a limitation of the approach used here.

There is an ever-present trade-off involved in choosing a framework to model uncertainty. In terms of applicability, realism, and simplicity, no one framework seems to be dominant in all aspects. Worse still, whichever framework is chosen, there are always assumed objects that simply must be regarded as hypothetical. For example, both the existence of constant acts for all outcomes, and the assumption that preferences are defined over all conceivable acts, even those counterfactual to any plausible reality, are highly questionable. One might claim that, if some aspects of each framework are necessarily hypothetical, then surely it does not matter if a hypothetical randomisation device is also used. Given this, it is reasonable to ask, at this point, does the choice of framework really matter? After all, subjective expected utility can be derived in all of the above frameworks, and this involves constructing objects with the same properties as a randomisation device. When we know it is not really necessary, the use of a hypothetical randomisation device that greatly simplifies the axiomatic work seems to be quite acceptable. For ambiguity models, further reassurance has been provided by Ghirardato, Maccheroni, Marinacci and Siniscalchi (2003), who show how many results developed with a hypothetical randomisation device can be recovered in the Wakker framework. However, their approach, based on a weak biseparability assumption, does not apply to variational preferences. Although a hypothetical randomisation device can be suitably defended, it would be concerning if a model simply could not be derived without such a device. This paper has addressed this concern for the variational preferences model. 


\section{Closing Comments}

This paper characterises variational preferences in a purely subjective framework. The key assumption was that, as in the Anscombe and Aumann (1963) approach, there are two stages of uncertainty. Even without the linear structure delivered by a randomisation device, multiple stages of uncertainty seem to be useful for utility measurement. ${ }^{6}$

Savage's expected utility axioms were extended to this framework, and axioms introduced: the second-stage sure-thing principle, second-stage uncertainty aversion, and second-stage constant-independence. It is not claimed here that this paper fully solves the open problem reported by Alon and Schmeidler (2014: 397). Progress is made here only by imposing additional conditions on the Savage (1954) framework.

\section{Acknowledgements}

I am grateful for the comments of an anonymous reviewer. The usual disclaimer applies.

\section{References}

[1] Abdellaoui, Mohammed, Aurelien Baillon, Laetitia Placido, and Peter P. Wakker (2011). The rich domain of uncertainty: Source functions and their experimental implementation. Amer Econ Rev 101, 695-723.

[2] Abdellaoui, Mohammed and Peter P. Wakker (2005). The likelihood method for decision under uncertainty. Theory Dec 58, 3-76.

[3] Alon, Shiri and David Schmeidler (2014). Purely subjective maxmin expected utility. J Econ Theory 152, 382-412.

\footnotetext{
${ }^{6}$ A similar idea forms the basis of conjoint measurement (Krantz, Luce, Suppes and Tversky, 1971).
} 
[4] Anscombe, Frank J. and Robert J. Aumann (1963). A definition of subjective probability. Ann Math Stat 34, 199-205.

[5] Casadesus-Masanell, Ramon, Peter Klibanoff, and Emre Ozdenoren (2000). Maxmin expected utility over Savage acts with a set of priors. J Econ Theory 92, 35-65.

[6] Camerer, Colin and Martin Weber. (1992). Recent developments in modeling preferences: Uncertainty and ambiguity. J Risk and Uncertainty 5, 325-370.

[7] Chateauneuf, Alain (1991). On the use of capacities in modeling uncertainty aversion and risk aversion. J Math Econ 22(6), 343-369.

[8] Cerreia-Vioglio, Simone, Fabio Maccheroni, Massimo Marinacci and Aldo Rustichini (2014). Niveloids and their extension: Risk measures on small domains. J Math Analysis and Applications 413, 343-360.

[9] Chew, Soo Hong and Jacob S. Sagi (2008). Small worlds: Modeling attitudes toward sources of uncertainty. J Econ Theory 139, 1-24.

[10] Chow, Clare Chua and Rakesh K. Sarin (2001). Comparative ignorance and the Ellsberg paradox. J Risk Uncertainty 22:2, 129-139.

[11] Ellsberg, Daniel (1961). Risk, ambiguity and the Savage axioms. QJE 75, 643-669.

[12] Ergin, Haluk and Faruk Gul (2009). A theory of subjective compound lotteries. J Econ Theory 144(3), 899-929.

[13] Fishburn, Peter C. (1970). Utility theory for decision making. Wiley, New York.

[14] Fox, Craig R. and Amos Tversky (1995). Ambiguity aversion and comparative ignorance. QJE 110, 585-603.

[15] Ghirardato, Paolo and Massimo Marinacci (2001). Risk, ambiguity, and the separation of utility and beliefs. Math Oper Res 26, 864-890. 
[16] Ghirardato, Paolo, Fabio Maccheroni, Massimo Marinacci and Marciano Siniscalchi (2003). A subjective spin on roulette wheels. Econometrica 71.6: 1897-1908.

[17] Gilboa, Itzhak (2014). Rationality and the Bayesian paradigm. J Econ Methodology, forthcoming.

[18] Gilboa, Itzhak and Massimo Marinacci (2013). Ambiguity and the Bayesian paradigm. In, Advances in Economics and Econometrics: Theory and Applications, Tenth World Congress of the Econometric Society. D. Acemoglu, M. Arellano, and E. Dekel (Eds.). New York, Cambridge.

[19] Gilboa, Itzhak, Andrew Postlewaite, and David Schmeidler (2012). Rationality of belief. Synthese 187, 11-31.

[20] Gilboa, Itzhak and David Schmeidler (1989). Maxmin expected utility with a nonunique prior. J Math Econ 18, 141-153.

[21] Hansen, L.P. and T.J. Sargent (2001). Robust control and model uncertainty. AER 91, 60-66.

[22] Halevy, Yoram (2007). Ellsberg revisited: an experimental study. Econometrica 75: 503-536.

[23] Heath, Chip and Amos Tversky (1991). Preference and belief: Ambiguity and competence in choice under uncertainty. J Risk Uncertainty 4, 5-28.

[24] Klibanoff, Peter, Massimo Marinacci, and Sujoy Mukerji (2005), A smooth model of decision making under uncertainy. Econometrica (6): 1849-1892.

[25] Krantz, David H., R. Duncan Luce, Patrick Suppes, and Amos Tversky (1971). Foundations of measurement, vol. I (Additive and polynomial representations). Academic Press, New York. 
[26] Machina, Mark J. (2011). Event-separability in the Ellsberg urn. Econ Theory 48, 425-436.

[27] Machina, Mark J. and David Schmeidler (1992). A more robust definition of subjective probability. Econometrica, 60, 745-780.

[28] Maccheroni, Fabio, Massimo Marinacci, and Aldo Rustichini (2006). Ambiguity aversion, robustness, and the variational representation of preferences. Econometrica $74,1447-1498$

[29] Nau, Robert (2006). Uncertainty aversion with second-order utilities and probabilities. Manag Sci 52, 136-145.

[30] Sarin, Rakesh K. and Peter P. Wakker (1997). A single-stage approach to Anscombe and Aumann's expected utility. Rev Econ Stud 64, 399-409.

[31] Savage, Leonard J. (1954). The foundation of statistics. Wiley, New York.

[32] Segal, Uzi (1987). The Ellsberg paradox and risk aversion: An anticipated utility approach. Int Econ Rev 28, 175-202.

[33] Seo, Kyoungwon (2009). Ambiguity and second-order belief. Econometrica 77, 1575-1605.

[34] Strzalecki, Tomasz (2011). Axiomatic foundations of multiplier preferences. Econometrica 79, 47-73.

[35] Trautmann, Stefan and Peter P. Wakker (2015). Making the Anscombe-Aumann approach suited for descriptive applications. Mimeo.

[36] Wakker, Peter P. (2001). Testing and characterizing properties of nonadditive measures through violations of the sure-thing principle. Econometrica 69, 1039-1059. 


\section{A Appendix}

\section{A.1 Proof of Theorem 1}

The necessity of the axioms follows from substituting the preference functional and elementary manipulations, hence the details are omitted here. We show the sufficiency of the axioms for the representation. The standard axioms for subjective expected utility hold for induced preferences over second-stage acts. Hence subjective expected utility holds over second-stage acts, for some utility $u$ and convex-valued probability measure $q$ over $\mathcal{E}_{\mathcal{T}}$. Let $U\left(f_{s}\right)=\int_{S_{\phi}} u\left(f_{s}(t)\right) d q(t)$ denote the subjective expected utility of a second-stage act. Let $\mathcal{S}=\left\{s_{1}, \ldots, s_{n}\right\}$. Let $U(f):=\left(U\left(f_{s_{1}}\right), \ldots, U\left(f_{s_{n}}\right)\right)$ and $U(\mathcal{A}):=\left\{U(f) \in \mathbb{R}^{n}: f \in\right.$

$\mathcal{A}\}$. For $\alpha \in[0,1]$ and acts $f, g \in \mathcal{A}$, define $\alpha U(f)+(1-\alpha) U(g)$ pointwise, so that:

$$
\alpha U(f)+(1-\alpha) U(g)=\left(\alpha U\left(f_{s_{1}}\right)+(1-\alpha) U\left(g_{s_{1}}\right), \ldots, \alpha U\left(f_{s_{n}}\right)+(1-\alpha) U\left(g_{s_{n}}\right)\right) .
$$

Note that, because $q$ is convex-valued, we are assured that $U(\mathcal{A})$ is a convex subset $\mathbb{R}^{n}$. By finiteness of $\mathcal{X}$, there is a best outcome $\bar{x}$ and worst outcome $\underline{x}$. By eventwise monotonicity, it can be shown that, for all $f \in \mathcal{A}, \bar{x} \geqslant f \geqslant \underline{x}$. Normalise $U$ so that $U(\bar{x})=1$ and $U(\underline{x})=0$. Consider an act $f \in \mathcal{A}$. By second-stage solvability there is a $A \in \mathcal{E}_{\mathcal{T}}$ such that $f \sim \bar{x}_{A} \underline{x}$. If $h=\bar{x}_{A} \underline{x}$, notice that that $U\left(h_{s}\right)=q(A)$ for all $s \in S$. Define utility function for acts $\phi: \mathbb{R}^{n} \rightarrow \mathbb{R}$ as follows: $\phi(U(f)):=\left\{U\left(h_{s}\right): f \sim \bar{x}_{A} \underline{x}=h\right\}$ for all $f \in \mathcal{A}$. Preferences $\geqslant$ over acts $\mathcal{A}$ are represented by the map $f \mapsto \phi(U(f))$.

We now show that $\phi$ satisfies three properties: monotonicity, mixture continuity, and vertical invariance. Call $\phi$ monotone if $U\left(f_{i}\right) \geqslant U\left(g_{i}\right)$ for all $i=1, \ldots, n$ implies $\phi(U(f)) \geqslant$ $\phi(U(g))$. Monotonicity of $\phi$ follows immediately from monotonicity, axiom 2. If, for all acts $f, g, h \in \mathcal{A}$, the sets $\{\alpha \in[0,1]: \phi(\alpha U(f)+(1-\alpha) U(g)) \geqslant \phi(U(h))\}$ and $\{\alpha \in[0,1]: \phi(\alpha U(f)+(1-\alpha) U(g)) \leqslant \phi(U(h))\}$ are closed in $[0,1]$, then $\phi$ is 
mixture continuous. The derived $U$ is known to be mixture continuous ${ }^{7}$ in that, for all $f, g, h \in \mathcal{A}$, the sets $\{\alpha \in[0,1]: \alpha U(f)+(1-\alpha) U(g) \geqslant U(h)\}$ and $\{\alpha \in[0,1]:$ $\alpha U(f)+(1-\alpha) U(g) \leqslant U(h)\}$ are closed in $[0,1]$. Then, by monotonicity, $\phi$ inherits mixture continuity.

If, for all acts $f, g \in \mathcal{A}$, first-stage constant act $h \in \mathcal{A}$ and $\alpha \in[0,1]$, we have $\phi(\alpha U(f)+$ $(1-\alpha) U(h))=\phi(\alpha U(f))+(1-\alpha) \phi(U(h))$, then $\phi$ is vertically invariant. An act $h \in \mathcal{A}$ is first-stage constant if, for all $s, \tilde{s} \in S$, the second-stage acts $h_{s}$ and $h_{\tilde{s}}$ coincide. Consider any act $f$ and first-stage constant act $h, f, h \in \mathcal{A}$ and event $A$ such that $q(A)=\alpha$, where $q$ is the subjective probability measure obtained above. Using solvability, it can be shown that there exists a first-stage constant act $g$ such that $f_{A} h \sim g_{A} h$. This holds if and only if:

$$
\phi\left(U\left(f_{A} h\right)\right)=\phi(\alpha U(f)+(1-\alpha) U(h))=\phi\left(U\left(g_{A} h\right)\right)=\phi(\alpha U(g)+(1-\alpha) U(h)) .
$$

Because $g$ and $h$ are first-stage constant, $g_{A} h$ is first-stage constant and, letting $\tilde{f}=g_{A} h$,

$$
\phi\left(U\left(g_{A} h\right)\right)=U\left(\tilde{f}_{s}\right)=\alpha U\left(g_{s}\right)+(1-\alpha) U\left(h_{s}\right)=\alpha \phi(U(g))+(1-\alpha) \phi(U(h)) .
$$

By the second-stage sure-thing principle, axiom $6, f_{A} h \sim g_{A} h$ only if $f_{A} \underline{x} \sim g_{A} \underline{x}$, which holds if and only if (recall $U(\underline{x})=0$ ):

$$
\phi\left(U\left(f_{A} \underline{x}\right)\right)=\phi(\alpha U(f))=\phi\left(U\left(g_{A} \underline{x}\right)\right)=\phi(\alpha U(g))=\alpha \phi(U(g)) .
$$

Therefore, collecting the above results, $\phi(\alpha U(f)+(1-\alpha) U(h))=\phi(\alpha U(f))+(1-$ $\alpha) \phi(U(h))$, hence $\phi$ is vertically invariant. This completes the proof of Theorem 1 .

\footnotetext{
${ }^{7}$ See, for example, Machina and Schmeidler (1992: 774-775).
} 


\section{A.2 Proof of Theorem 2}

Axioms 1-6 have been shown, in Theorem 1, to be equivalent to preferences admitting an invariant second-stage expected utility representation. If, for all acts $f, g \in \mathcal{A}$ and $\alpha \in[0,1]$ we have $\phi(\alpha U(f)+(1-\alpha) U(g)) \geqslant \alpha \phi(U(f))+(1-\alpha) \phi(U(g))$, then $\phi$ is concave. We show that second-stage uncertainty aversion is equivalent to concavity of $\phi$.

Take any acts $f, g \in \mathcal{A}$ with $f \sim g$, so that $\phi(U(f))=\phi(U(g))$. By second-stage solvability, there exists $A(s)$ such that $f_{s} \sim\left(\bar{x}_{A(s)} \underline{x}\right)_{s}$ and there exists $C(s)$ such that $f_{s} \sim$ $\left(\bar{x}_{C(s) \underline{x}}\right)_{s}$, for all $s \in \mathcal{S}$. This holds if and only if $U\left(f_{s}\right)=q(A(s))$ and $U\left(g_{s}\right)=q(C(s))$ for all $s \in \mathcal{S}$. Because $q$ is convex-valued we can find, for all $s \in \mathcal{S}$, second-stage events $B(s)$ such that $q(B(s))=\frac{1}{2} q(A(s))+\frac{1}{2} q(C(s))$. Notice that, for all $s \in S, B(s)$ is a secondstage event average of $A(s)$ and $C(s)$. Then, an act $h$ with $h_{s}=\left(\bar{x}_{B(s)} \underline{x}\right)_{s}$ for all $s \in S$ is a second-stage act average of $f$ and $g$ with $U(h)=\frac{1}{2} U(f)+\frac{1}{2} U(g)$. By second-stage uncertainty aversion, axiom $7, h \geqslant f$, which holds if and only if $\phi(U(h))=\phi\left(\frac{1}{2} U(f)+\right.$ $\left.\frac{1}{2} U(g)\right) \geqslant \phi(U(f))$. Let $\mathcal{D}$ be the set of dyadic rationals. Applying the above finitely many times, it can be shown that, for all $\alpha \in[0,1] \cap \mathcal{D}, \phi(U(f))=\phi(U(g))$ implies $\phi(\alpha U(f)+(1-\alpha) U(g)) \geqslant \phi(U(f))$. $\phi$ is mixture continuous, hence $\phi$ is quasi-concave. By Lemma 25 of MMR, $\phi$ is concave (see also Theorem 4 of Cerreia-Vioglio, Maccheroni, Marinacci and Rustichini (2014)). By Lemma 26 of Maccheroni, Marinacci and Rustichini (2006: 1476-1477), $\phi$ has the following representation:

$$
\phi(U(f))=\min _{p \in \mathcal{P}_{S}}\left(\int_{S} U(f) d p(s)+c(p)\right)=\min _{p \in \mathcal{P}_{S}}\left(\int_{S} \int_{\mathcal{T}} u(f(s, t)) d p(s) d q(t)+c(p)\right)
$$

where $u: \mathcal{X} \rightarrow \mathbb{R}$ is a strictly $\geqslant$-increasing utility function and $c: \mathcal{P}_{S} \rightarrow[0, \infty]$ is a grounded, convex, and lower semicontinuous function. Therefore, preferences over $\mathcal{A}$ satisfying the axioms of statement 1 of Theorem 2 are variational preferences. For the uniqueness results, cardinality of $u$ and uniqueness of $q$ are well-known. That $c$ is a ratio scale follows from MMR's corollary 5. 


\section{A.3 Proof of Theorem 3}

If preferences satisfy axioms 1-6, then, following the proof of Theorem 2, preferences are represented by a functional $f \mapsto \phi(U(f))$. We have established that $\phi$ is monotonic, mixture continuous and vertically invariant, and $\phi$ is concave if and only if preferences satisfy second-stage uncertainty aversion. Now assume certainty equivalents exist for all acts. Fix $U(\bar{x})=1$ and $U(\underline{x})=0$. Take any $a=\left(a_{1}, \ldots, a_{n}\right)$ and $b=\left(b_{1}, \ldots, b_{n}\right)$, with $a, b \in[0,1]^{n}$. For all $a_{i}, b_{i}, i=1, \ldots, n$, by convex-valuedness of $q$, there exist events $A_{i}$ and $B_{i}$ with $q\left(A_{i}\right)=a_{i}$ and $q\left(B_{i}\right)=b_{i}$. Let $x(E)$ denote the certainty equivalent of $\bar{x}_{E} \underline{x}$, for $E \in \mathcal{E}_{\mathcal{T}}$. Then, the act $f$ with $f_{s_{i}}=x\left(A_{i}\right)$ for all $i=1, \ldots, n$ has utility vector $U(f)=a$, and the act $g$ with $g_{s_{i}}=x\left(B_{i}\right)$ for all $i=1, \ldots, n$ has utility vector $U(g)=b$. Notice that $f$ and $g$ are second-stage constant acts. By convex-valuedness of $q$, there exists a second-stage event $C$ with $q(C)=\frac{1}{2}$. Then, the act $f_{C} g$ generates utility vector $U\left(f_{C} g\right)=\frac{1}{2} a+\frac{1}{2} b$. Second-order risk aversion holds, hence $f \sim g$ only if $f_{C} g \geqslant f$. Equivalently: $\phi\left(U\left(f_{C} g\right)\right)=\phi\left(\frac{1}{2} a+\frac{1}{2} b\right) \geqslant \phi(U(f))=\phi(a)$. Because $\phi$ is mixture continuous, $\phi$ is quasi-concave. Monotonicity and vertical invariance have been shown. Hence, under axioms 1-6 and existence of certainty equivalents, second-order risk aversion holds if and only if $\phi$ is concave.

\section{A.4 Proof of Theorem 4}

The necessity of the axioms involves only substitution of the preference representation. We prove the sufficiency of the axioms for the representation. Axioms 1-7 hold, hence preferences admit a representation $f \mapsto \phi(U(f))$, with $\phi$ monotonic, mixture continuous, vertically invariant and concave. Second-stage constant independence allows us to establish that $\phi$ is linearly homogeneous: For all $f \in \mathcal{A}$ and $\alpha \in[0,1]$ we have $\phi(\alpha U(f))=\alpha \phi(U(f))$. Let $f \in \mathcal{A}$ be an act and let $g$ be a first-stage constant act such that $f \sim g$, or equivalently $\phi(U(f))=\phi(U(g))$. There exist acts $\tilde{f}$ and $\tilde{g}$ such that $\tilde{f}$ is a second-stage act average of 
$f$ and $\underline{x}$ and $\tilde{g}$ is a second-stage act average of $g$ and $\underline{x}$. The second-stage constant independence axiom implies $\tilde{f} \sim \tilde{g}$, or equivalently $\phi\left(\frac{1}{2} U(f)\right)=\phi\left(\frac{1}{2} U(g)\right)=\frac{1}{2} \phi(U(g))$, where the second equality exploits that $g$ is first-stage constant. Hence, $\phi\left(\frac{1}{2} U(f)\right)=\frac{1}{2} \phi(U(f))$. The same technique can be used iteratively to show that $\phi(\alpha U(f))=\alpha \phi(U(f))$ for all $\alpha \in[0,1] \cap \mathcal{D}$. Linear homogeneity follows as $\phi$ is mixture continuous. It follows from Lemma 3.5 of Gilboa and Schmeidler (1989) that $\phi$ is a subjective maxmin expected utility representation. 